\title{
Transverse optical forces for manipulating nanoparticles
}

\author{
Alexander A. Zharov and Alexander A. Zharov, Jr. \\ Institute for Physics of Microstructures, Russian Academy of Sciences, Nizhny Novgorod 603950, Russia \\ and N.I. Lobachevsky State University, Nizhny Novgorod 603950, Russia
}

Ilya V. Shadrivov

Nonlinear Physics Centre, Research School of Physics and Engineering, The Australian National University, Canberra ACT 2601, Australia

Nina A. Zharova*

Institute of Applied Physics, Russian Academy of Sciences, Nizhny Novgorod 603950, Russia

(Received 11 March 2016; published 20 December 2016)

\begin{abstract}
We study optical forces acting on a subwavelength particle with anisotropic polarizability and discover an optomechanical effect that resembles the Hall effect for electrons. While in the classical Hall effect the transverse Lorentz force and the transverse voltage appear due to the static magnetic field which induces the nondiagonal components of the electric conductivity tensor; in our case the imaginary parts of the nondiagonal elements of the polarizability tensor are responsible for the transverse scattering force. We calculate this force for the examples of the ellipsoidal plasmonic nanoparticles and the spherical particle with gyromagnetic properties, and show that the transverse force depends on the physical origin of the anisotropy of the polarizability, and on the electromagnetic wave structure around the particle. Moreover, this force primarily occurs in the inhomogeneous field only.
\end{abstract}

DOI: 10.1103/PhysRevA.94.063845

\section{INTRODUCTION}

Mechanical manipulation of nano- and microparticles by light is one of the most fascinating phenomena studied by modern nanophotonics, and it is already used in several applications. Probably the first demonstration of the control of dielectric microspheres using a laser is presented by Ashkin in 1970 [1]. Nowadays, the manipulation of particles by light attracts significant interest, and it is strongly motivated by applications such as optical tweezers, which are used for moving biological objects, including cells, DNA, or big organic molecules [2-5]. Apart from biological and medical applications, light can be used for sorting, moving, and separating dielectric or metal nanoparticles, which is extremely important for mass and size spectrometry of nanoparticle mixtures [6-11].

In general, for calculating optical forces one can use Maxwell's stress tensor [12], and for complex structures this can be done only numerically. Purely numerical results are often quite obscure; therefore analytical models describing simple problems are very valuable, since they can give a deeper physical insight. One of the most popular objects of analytical studies is a spherical particle made of a homogeneous isotropic material, illuminated by an optical wave of a simple structure, e.g., a linearly polarized plane wave or a Gaussian beam. In such simple cases we can calculate all the forces acting on small particles analytically. It is not surprising then that as a result of such simplified models a number of new effects for more complex particles, or for more complex optical beams, still remain undiscovered.

The optical forces acting on particles can be attributed to one of the two types-either gradient or scattering [13,14]. The gradient force is proportional to the gradient of the field

\footnotetext{
*zhani@appl.sci-nnov.ru
}

intensity as well as to the real part of the particle polarizability, and it is a generalization of the static electrophoretic force [15], though it includes both electric and magnetic parts of the Lorentz force. This is the force that is used in optical tweezers for manipulation of nanoparticles, and it requires structured optical beams. The scattering force is proportional to the imaginary part of the electric polarizability, and it is directed along the wave vector of the electromagnetic wave, because this force appears as a result of the momentum transfer from the absorbed or scattered photons to the particle.

In this paper, we study the interaction of light with several types of anisotropic subwavelength nanoparticles. In the simplest dipole approximation we show that the average scattering force acquires a component in the direction orthogonal to the wave vector. This force is proportional to the imaginary part of the nondiagonal component of the polarizability tensor of the particle and it resembles the electronic Hall effect (see Fig. 1). Here the role of charged electrons is played by neutral photons, and instead of transverse voltage induced by Lorentz force there is a transverse component of the optical scattering force.

We further show that the anisotropy of polarizability that leads to the transverse force may be caused by various reasons: it can be an anisotropic nature of the material that makes up a spherical particle, it can be the shape of the particle that induces the anisotropy, or the inhomogeneity of the material that the particle is made of. A similar effect was recently studied [16] where a lateral recoil force emerges due to the directional surface plasmon excitation by a nanoparticle placed near the air/metal interface and illuminated by a circularly polarized light.

\section{OPTICAL FORCES IN THE FIELD OF INHOMOGENEOUS PLANE WAVES}

We start with deriving expressions for the optical forces acting on a nanoparticle in a TM-polarized inhomogeneous 


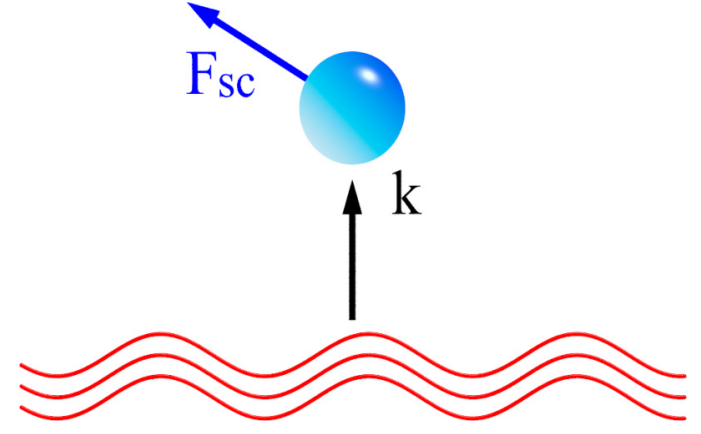

FIG. 1. Schematics of the problem. Inhomogeneous plane wave, e.g., created by interference of two plane waves or just a surface wave, is incident on a particle that has anizotropic properties. The wave scatters on the particle and in certain conditions it creates scattering optical force with a finite component orthogonal to a wave vector.

plane wave. We assume that the incident wave has the following field structure:

$$
\begin{aligned}
& H_{y}=A(z) \exp (-i k x), \\
& E_{x}=-\frac{1}{i k_{0} \varepsilon} \frac{d A(z)}{d z} \exp (-i k x), \\
& E_{z}=-\frac{k}{k_{0} \varepsilon} A(z) \exp (-i k x),
\end{aligned}
$$

where the real function $A(z)$ satisfies the Helmholtz equation,

$$
\frac{d^{2} A}{d z^{2}}+\left(k_{0}^{2} \varepsilon-k^{2}\right) A=0,
$$

and describes the transverse field structure, $\varepsilon$ is the dielectric permittivity of the environment, and $k_{0}=\omega / c$ is the wave number in free space, $\omega$ is the angular frequency, and $c$ is the speed of light. A small dipole particle with anisotropic polarizability $\alpha_{i k}$ placed in such an electromagnetic wave experiences the ponderomotive force

$$
\begin{aligned}
\mathbf{F} & =\left\langle(\mathbf{p} \nabla) \mathbf{E}+\frac{1}{c}\left[\frac{\partial \mathbf{p}}{\partial t} \times \mathbf{B}\right]\right\rangle \\
& =\frac{1}{2}\left[(\mathbf{p} \nabla) \mathbf{E}^{*}+\left[\mathbf{p} \times \operatorname{curl} l \mathbf{E}^{*}\right]+\text { c.c. }\right],
\end{aligned}
$$

where $\langle\ldots\rangle$ stands for time averaging. The expression for the force can be rewritten as

$$
F_{i}=\frac{1}{2} \sum_{k}\left(p_{k} \nabla_{i} E_{k}^{*}+\text { c.c. }\right),
$$

where $p_{k}=\sum_{j} \alpha_{k j} E_{j}$ is the dipole moment of the particle, and the star denotes complex conjugation. In the case of the TM-polarized wave described by the expressions (1), the force components (4) can be written explicitly as

$$
\begin{gathered}
F_{x}^{s c}=-\frac{k}{\left(k_{0} \varepsilon\right)^{2}}\left[\operatorname{Im} \alpha_{x x}\left(\frac{d A}{d z}\right)^{2}+k^{2} \operatorname{Im} \alpha_{z z} A^{2}\right], \\
F_{z}=F_{z}^{\mathrm{grad}}+F_{z}^{s c},
\end{gathered}
$$

where

$$
\begin{gathered}
F_{z}^{\mathrm{grad}}=\frac{1}{2\left(k_{0} \varepsilon\right)^{2}}\left[k^{2} \operatorname{Re} \alpha_{z z}-\left(k_{0}^{2} \varepsilon-k^{2}\right) \operatorname{Re} \alpha_{x x}\right] \frac{d A^{2}}{d z}, \\
F_{z}^{s c}=\frac{k}{\left(k_{0} \varepsilon\right)^{2}}\left[\left(k_{0}^{2} \varepsilon-k^{2}\right) \operatorname{Im} \alpha_{x z} A^{2}+\operatorname{Im} \alpha_{z x}\left(\frac{d A}{d z}\right)^{2}\right],
\end{gathered}
$$

and Re and Im stand for real and imaginary parts, respectively.

We see that the transverse component of the force $F_{z}$, in addition to the usual gradient part, also contains a scattering force component which is proportional to the imaginary part of the polarizability tensor. As we mentioned earlier, the scattering force is caused by the momentum transfer from absorbed and/or scattered photons to the nanoparticle. From first glance, one can expect that this force should be parallel to the wave momentum, i.e., along the wave vector $\mathbf{k}$, since the nanoparticle scatters as a dipole, and the dipole scattering is always symmetric, independently of the polarizability tensor. However, if we can break the cylindrical symmetry of the scattering pattern, then we can gain net force orthogonal to the momentum of incoming light.

Below we give particular examples with a clear explanation of such an asymmetric behavior of an apparently symmetric system. We consider two cases when the anisotropy has two different origins: a plasmonic metallic nanoparticle with the anisotropic shape of an ellipsoid of revolution and a spherical gyrotropic ferromagnetic nanoparticle.

\section{ELLIPTIC PLASMONIC NANOPARTICLES}

First, we study plasmonic metallic nanoparticles that have the shape of an ellipsoid of revolution. The polarizability tensor for the subwavelength ellipsoids ${ }^{1}$ are known [17], and the relevant components can be written in the following form:

$$
\begin{gathered}
\alpha_{x x}=\alpha^{\perp}+\left(\alpha^{(\|)}-\alpha^{(\perp)}\right) \times \cos ^{2} \varphi \sin ^{2} \theta, \\
\alpha_{z z}=\alpha^{\perp}+\left(\alpha^{(\|)}-\alpha^{(\perp)}\right) \times \cos ^{2} \theta, \\
\alpha_{x z}=\alpha_{z x}=\left(\alpha^{(\|)}-\alpha^{(\perp)}\right) \times \cos \varphi \sin \theta \cos \theta,
\end{gathered}
$$

where the spherical angles $\varphi, \theta$ determine orientation of the ellipsoid in space relative to our coordinate frame. $\alpha^{(\|)}$and $\alpha^{(\perp)}$ are longitudinal and transverse parts of the polarizability tensor in the principal axis of the ellipsoid of revolution, which are given by [17]

$$
\begin{aligned}
\alpha^{(\perp)} & =\frac{a^{2} b}{3} \frac{\varepsilon_{m}(\omega)-\varepsilon}{\varepsilon+1 / 2\left(\varepsilon_{m}(\omega)-\varepsilon\right)(1-n)}, \\
\alpha^{(\|)} & =\frac{a^{2} b}{3} \frac{\varepsilon_{m}(\omega)-\varepsilon}{\varepsilon+\left(\varepsilon_{m}(\omega)-\varepsilon\right) n} .
\end{aligned}
$$

Here $a$ and $b$ represent principal semiaxes of the ellipsoid, $\varepsilon_{m}(\omega)$ is the frequency-dependent particle permittivity, and $n$

\footnotetext{
${ }^{1}$ We use an electrostatic expression for the polarizability and do not take into account radiation corrections whose impact in our case is small compared to that of the dissipation.
} 
is the depolarization factor

$$
n=\frac{1}{2}\left(\frac{a}{b}\right)^{2} \int_{0}^{\infty} \frac{d x}{(1+x)\left[x+(a / b)^{2}\right]} .
$$

The depolarization factor assumes values in the range $0<n<$ 1 , with $n=1 / 3$ corresponding to a sphere, while the values $0<n<1 / 3$ relate to elongated particles and $1 / 3<n<1$ correspond to oblate ellipsoids. The difference $\alpha^{(\|)}-\alpha^{(\perp)}$ vanishes only in the case of a spherical particle, which leads to the isotropic polarizability and obvious lack of transverse scattering force.

As a first particular example, we consider the field created by two TM-polarized plane waves of the same amplitude symmetrically converging at an angle $2 \beta, 0<\beta<\pi / 2$ that, for example, corresponds to the TM mode structure in a planar waveguide. The magnetic field of such wave can be represented in the form given by Eq. (1) with

$$
A(z)=\frac{1}{2}\left(A_{m} e^{-i \varkappa z}+A_{m} e^{i \varkappa z}\right)=A_{m} \cos \varkappa z,
$$

where $k=k_{0} \sqrt{\varepsilon} \cos \beta$ and $\varkappa=k_{0} \sqrt{\varepsilon} \sin \beta$. This field has a standing wave pattern in $z$ direction and traveling wave structure along $x$, and it is perfectly symmetric with respect to the $z=0$ plane. However, in this case the substitution of expression (14) into Eq. (8), for the symmetric polarizability tensor of plasmonic particles $\left(\alpha_{i k}=\alpha_{k i}\right)$, yields

$$
F_{z}^{(s c)}=\frac{k \varkappa^{2}}{\left(k_{0} \varepsilon\right)^{2}} A_{m}^{2} \operatorname{Im}\left(\alpha_{x z}\right)=\text { const. }
$$

This expression describes a nonvanishing transverse scattering force, which is independent of the $z$ coordinate. This result can be intuitively explained in terms of the action of partial plane waves on the anisotropic particle. Due to the nondiagonal component of polarizability, each plane wave in expression (14) produces different scattering forces acting on the nanoparticle along its own wave vector, and this produces a nonzero component of the scattering force in the $z$ direction.

For further explanation of the effect, we study the scattering pattern of the nanoparticle. Despite the symmetric scattering pattern of a dipole in the field of a homogeneous plane wave, in the case of an incident inhomogeneous plane wave the interference of the field scattered by the dipole with partial converging homogeneous plane waves leads to the asymmetry of the scattering that, in turn, produces the nonzero radiation reaction force along $z$.

To further confirm our findings, we use the finite-element method to numerically calculate the scattering of light by a small silver elliptic particle. As an example, we analyze the scattering characteristics of an axially symmetric elongated particle with the transverse and longitudinal semiaxes $a=$ $15 \mathrm{~nm}$ and $b=30 \mathrm{~nm}$, placed in vacuum. The depolarization factor for this particle is $n=0.1677$. Figure 2 shows the distribution of the electric field (amplitude in log scale) in the vicinity of this particle, when the particle is placed in the maximum of the standing wave given by Eqs. (1) and (14). The wavelength in our calculations is $\lambda=500 \mathrm{~nm}$, the angle is $\beta=54.7^{\circ}$, and the amplitude $A_{m}=27.46 \mathrm{~V} / \mathrm{cm}$. This amplitude of the field corresponds to the mean power flow density of $1 \mathrm{~W} / \mathrm{cm}^{2}$ in the plane-wave regime (when $\beta=0$ ). The field structure is shown for two cases: symmetric,
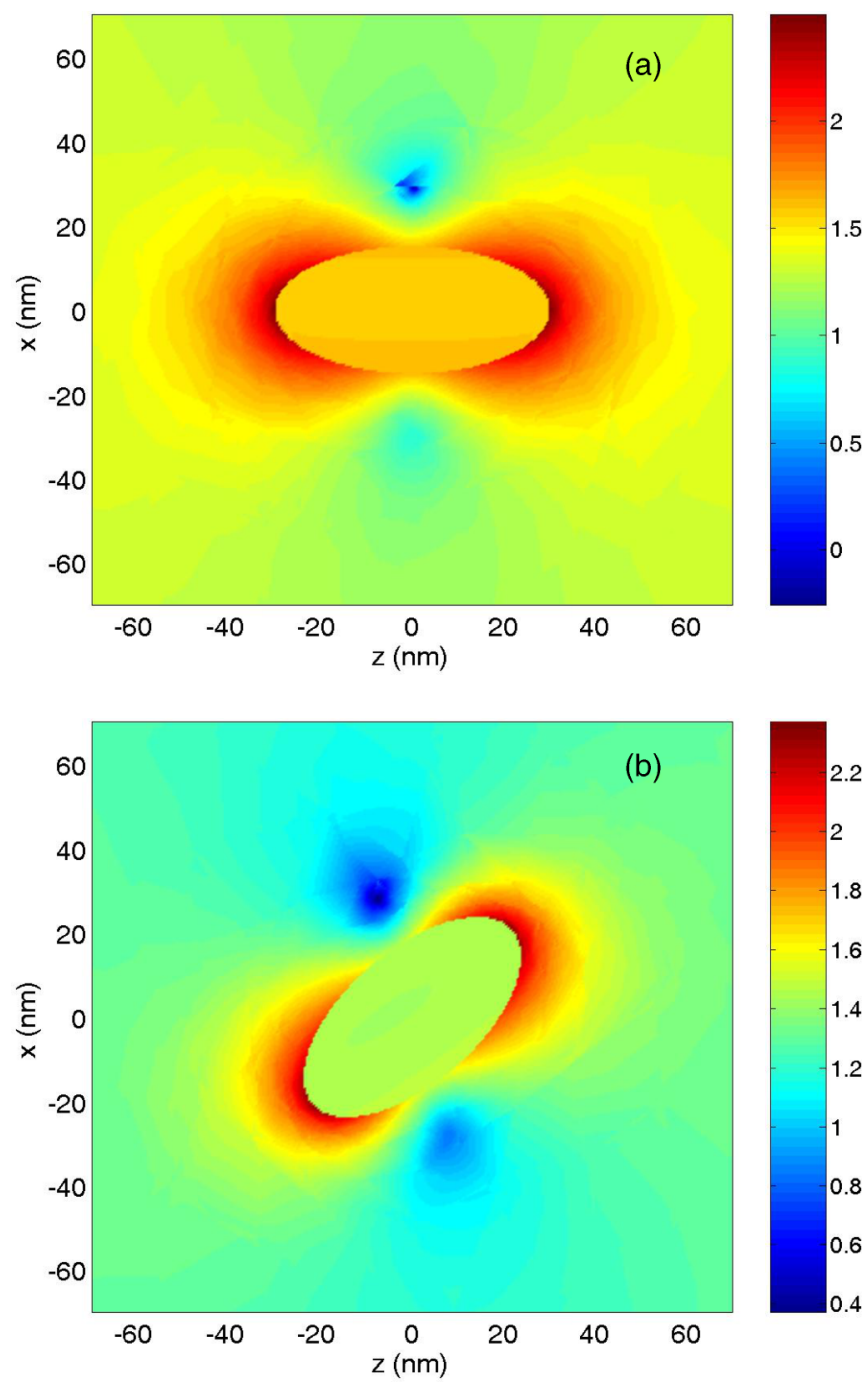

FIG. 2. Electric field distribution (shown is the amplitude of electric field in $\mathrm{V} / \mathrm{m}$ in logarithmic scale for the incident plane wave with energy flow of $1 \mathrm{~W} / \mathrm{cm}^{2}$ ) on the $x z$ plane in the vicinity of the elliptic silver particle placed in the origin (a) symmetric case with the ellipsoid oriented along the $z$ axis and (b) the long axis of the particle is at $45^{\circ}$ to the $z$ axis. Parameters of the particle and the incident field are given in the text.

when the ellipsoid is oriented along the $z$ axis, and a more general case with broken symmetry when the long axis of the particle makes the angle $\theta=45^{\circ}$ with the $z$ axis.

The characteristic angular dependence of the optomechanical Hall force can be derived from Eq. (15) as

$$
F_{z}^{(s c)} \sim \cos \beta \sin ^{2} \beta \cos \varphi \sin \theta \cos \theta,
$$

which gives the optimal convergence angle $\beta \approx 54.7^{\circ}$ $(\cos \beta=1 / \sqrt{3})$ that provides a maximum of the force. It is clear that transverse scattering force is absent in a homogeneous traveling wave $(\beta=0)$, or in a purely standing wave $(\beta=\pi / 2, k=0)$. It also vanishes in the case when the principal axis of the ellipsoid of revolution coincides with the wave-vector direction, i.e., $\varphi=\pi / 2$, or $\theta=0$, or $\theta=\pi / 2$. Also, $F_{z}^{(s c)}=0$ in the field of crossing TE-polarized electromagnetic waves because of the absence of the longitudinal 


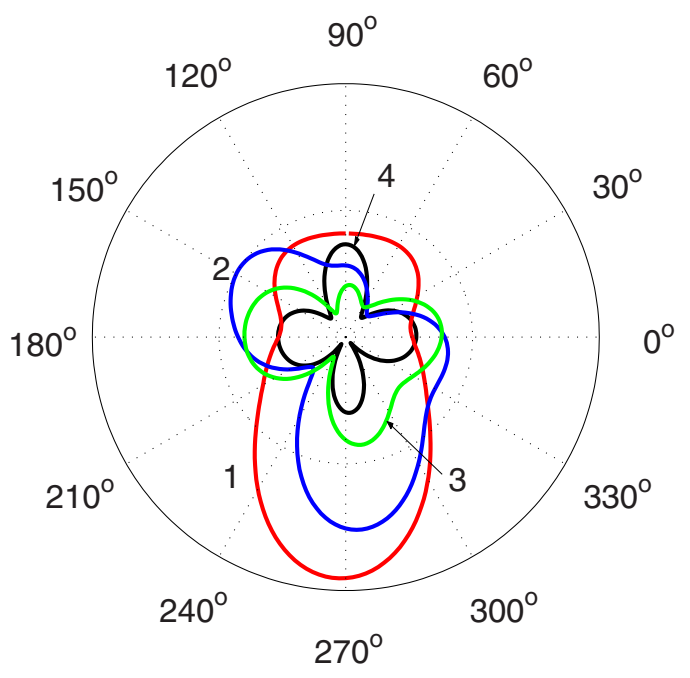

FIG. 3. Scattering diagram in the $x z$ plane for four orientations of the ellipsoidal silver nanoparticle placed in the origin $\theta=0^{\circ}(1)$, $\theta=30^{\circ}(2), \theta=60^{\circ}(3)$, and $\theta=90^{\circ}$ (4). Other parameters are the same as in Fig. 2.

(along $x$ ) component of the electric field. In the latter case only a gradient transverse force appears.

Figure 3 shows a scattering diagram in the $x z$ plane for four different orientations of the particle, $\theta=0^{\circ}, 30^{\circ}, 60^{\circ}, 90^{\circ}$.

The total optical force experienced by a small scatterer can be calculated using Ref. [18],

$$
\langle\mathbf{F}\rangle=-\frac{\sqrt{\varepsilon}}{c} \oint\left(\langle\mathbf{S}\rangle-\left\langle\mathbf{S}_{\mathbf{i}}\right\rangle\right) d s,
$$

where integration is performed over the closed surface in far field and $\mathbf{S}, \mathbf{S}_{\mathbf{i}}$ are the total Poynting vector and the Poynting vector of the incident wave, respectively. From this equation we conclude that an asymmetric scattering pattern (such as curves 2 and 3 in Fig. 3) corresponds to a nonzero transverse force experienced by the particle. The symmetric case of curves 1 and 4 in this figure corresponds to $\left\langle\mathbf{F}_{\mathbf{z}}\right\rangle \approx 0$. We note that the scattering radiation of a chiral particle can be asymmetric [19], also leading to the appearance of the transverse force. Direct force calculations based on the Maxwell's stress tensor give in our case $F_{z}^{(1)}=7.23 \times$ $10^{-24} \mathrm{~N}, F_{z}^{(2)}=-2.29 \times 10^{-20} \mathrm{~N}, F_{z}^{(3)}=-2.28 \times 10^{-20} \mathrm{~N}$, and $F_{z}^{(4)}=2.19 \times 10^{-23} \mathrm{~N}$ (the particle's volume is $v=$ $2.8 \times 10^{-17} \mathrm{~cm}^{3}$ and field intensity in the maximum of the standing wave is $0.58 \mathrm{~W} / \mathrm{cm}^{2}$ ).

Ellipsoidal particles have several plasmonic resonances, described by the zeros of the denominator of Eq. (12). For our particle, the lower resonance occurs at $\lambda \approx 448 \mathrm{~nm}$, while the next resonance is outside the visible frequency range. At the resonance both real and imaginary parts of the polarizability grow, thus increasing the total force experienced by the particle. Figure 4 shows both the longitudinal $F_{x}(\omega)$ and transverse gradient, $F_{z}^{(\text {grad }}(\omega)$, and scattering $F_{z}^{(s c)}(\omega)$ forces. To calculate the gradient force, we have evaluated the total transverse force at the point of maximum intensity gradient, $z=\pi / 4 \varkappa$, and subtracted the value of $F_{z}^{(s c)}$, which is equal to the total transverse force at $z=0$. Figure 4 also shows the

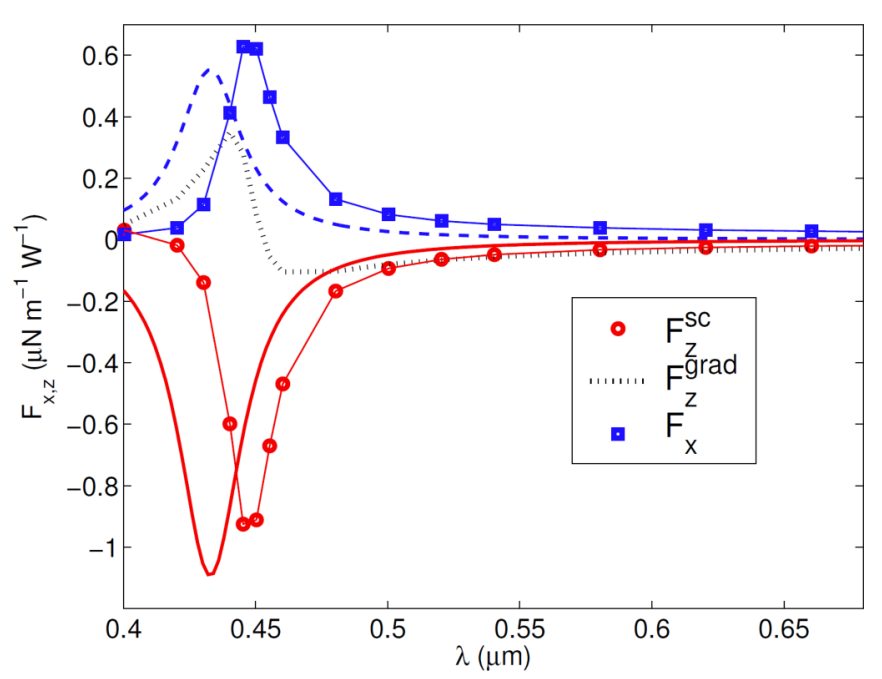

FIG. 4. Frequency dependence of the forces $F_{x}(\omega), F_{z}^{(\text {grad })}(\omega)$, and $F_{z}^{(s c)}(\omega)$. Red circles, black dotted line, and blue squares show numerically calculated $F_{z}^{(s c)}, F_{z}^{\text {(grad) }}$, and $F_{x}$. Solid red and dashed blue lines show analytically calculated forces, $f_{z}^{(s c)}$ and $f_{x}$.

forces calculated from a simple model theory [17]. Both in numerical simulations and model calculations the dispersion characteristics of silver were taken from [20]. Here and in what follows we present forces per unit volume calculated for the incident field intensity of $1 \mathrm{~W} / \mathrm{m}^{2}$. To obtain full physical force for arbitrary intensity, the calculated force per unit volume has to be multiplied by the volume of the particle and by the intensity of incident light. Both analytical $\mathbf{f}$ and numerical F forces demonstrate qualitatively similar resonant behavior, with the resonance frequency of full numerical results being redshifted as compared to the analytical results. This can be expected, since the analytical model [17] is quasistatic and it does not take into account the finite size of the particle.

Total transverse force consisting of the sum of the gradient and scattering forces may cause continuous drift of the particle. This occurs if the scattering force, which is constant in the considered field configuration, prevails over the gradient force, which is periodic along the $z$ axis. According to the results shown in Fig. 4, this is possible in the wavelength interval $430 \mathrm{~nm}<\lambda<510 \mathrm{~nm}$, i.e., near the resonance. This frequency range can be efficiently controlled by an appropriate choice of the convergence angle of the two plane waves $\beta$ and it is shown by shaded areas in Fig. 5 for two different angles $\theta$.

It is interesting to note that the transverse scattering force strongly depends on the external field structure. A specific example is the nanoparticle in the plane inhomogeneous wave with purely exponential transverse structure

$$
A(z)=A_{m} \exp (-\varkappa z), \quad z>0,
$$

with $\varkappa^{2}=k^{2}-k_{0}^{2} \varepsilon$. This field structure may correspond to a surface wave or represent the field beyond the reflecting edge of the prism at the total internal reflection. Since surface waves are slower than the speed of light, then $\varkappa$ in Eq. (17) is purely real. Using Eq. (8) we derive

$$
F_{z}^{(s c)}=\frac{k}{\left(k_{0} \varepsilon\right)^{2}} \operatorname{Im} \alpha_{x z}\left[-\varkappa^{2} A_{m}^{2} e^{-2 \varkappa z}+\left(-\varkappa A_{m} e^{-\varkappa z}\right)^{2}\right]=0,
$$




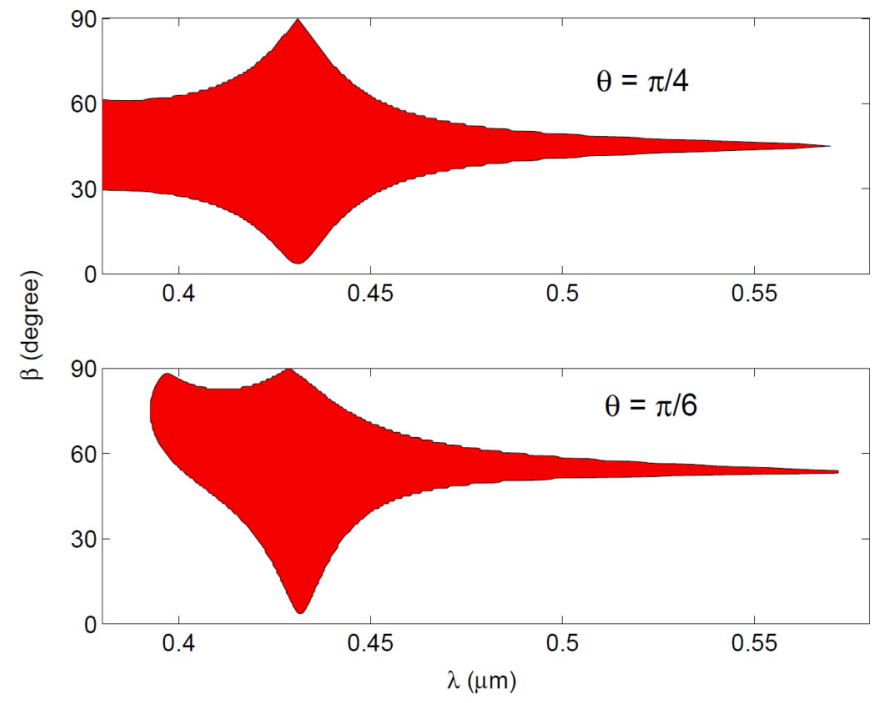

FIG. 5. Areas of continuous drift (red) in the $\beta-\lambda$ plane for $\theta=$ $\pi / 4$ and $\theta=\pi / 6$. The factor $a / b=1 / 2(n=0.1677)$.

meaning that the transverse scattering force for plasmonic nanoparticles does not exist in the field of the evanescent surface waves.

\section{FORCES ACTING ON GYROTROPIC PARTICLES}

As a second example, we examine the spherical gyrotropic ferromagnetic nanoparticle with the magnetization axis directed parallel to the $y$ axis. Orientation of the magnetization axis can be controlled by an external static magnetic field. The polarizability tensor of such a particle can approximately be written in the optical domain as [21]

$$
\hat{\alpha}=\left(\begin{array}{ccc}
\alpha & 0 & i u \\
0 & \alpha & 0 \\
-i u & 0 & \alpha
\end{array}\right)
$$

where $\alpha \approx a^{3}\left(\varepsilon_{m}-\varepsilon\right) /\left(\varepsilon_{m}+2 \varepsilon\right), u=3 g a^{3} /\left(\varepsilon_{m}+2 \varepsilon\right)^{2}$, and $g$ is the gyrotropy parameter, which is proportional to the magnetization.

We consider the lossless case, so the tensor $\hat{\alpha}$ is Hermitian. We also take into account that in the optical frequency range the diagonal components of the polarizability tensor are approximately equal. The scattering force, however, can still appear in certain fields because of the different signs of the nondiagonal components of the polarizability tensor for gyrotropic materials. In the field of two converging plane waves, Eq. (14), the scattering force component, which is perpendicular to the wave vector, is given by

$$
F_{z}^{(s c)}=\frac{k}{\left(4 k_{0} \varepsilon\right)^{2}} A_{m}^{2} \varkappa^{2} u \cos 2 \varkappa z,
$$

and in the case of a surfacelike wave, Eq. (17), the force is also nonzero and it can be written in the form

$$
F_{z}^{(s c)}=-\frac{2 k}{\left(k_{0} \varepsilon\right)^{2}} A_{m}^{2} u \varkappa^{2} \exp (-2 \varkappa z) .
$$

Since dipole radiation is symmetric, a nonzero transverse force can be provided only by asymmetric destructive and constructive interference of scattered and incident waves and it induces the momentum along $z$, which, in turn, leads to the scattering force along $z$. We note that the optical transverse force acting on the gyrotropic ferromagnetic nanoparticle in the optical exponential field is small compared to the gradient force even at plasmonic resonances, $F_{z}^{c s} / F_{z}^{g r} \sim 3 g /\left(\varepsilon_{m}-\right.$ $\varepsilon)\left(\varepsilon_{m}+2 \varepsilon\right)$, due to the small values of gyrotropy parameters in available materials $g \sim 10^{-3}$.

We note that in this work we study time-averaged forces acting on nanoparticles. However, the incident field can induce not only force, but also a time-averaged torque which reorients particles and therefore suppresses the considered effect. In practice, one can apply a static electric field that will keep particle orientation via electrophoretic forces.

\section{v. CONCLUSIONS}

To conclude, we predict an optomechanical effect which manifests itself as a transverse electromagnetic scattering force acting on an anisotropic nanoparticle in the direction perpendicular to the wave vector of an incident TM-polarized inhomogeneous plane wave. This force is proportional to the imaginary part of the nondiagonal component of the polarizability tensor of the particle. In the case of a gyrotropic particle, the off-diagonal terms in the polarizability are caused by the magnetic field, and this creates a close analogy to a classical electronic Hall effect, when a transverse force can be induced by a magnetic field perpendicular to electric current. While in the electronic Hall effect the magnetic field induces nondiagonal components of the conductivity tensor, in optics the magnetic field can induce the nondiagonal elements of the particle polarizability. We also show that the predicted optomechanical effect is more general, and it may occur in other cases when the particle polarizability has off-diagonal terms.

\section{ACKNOWLEDGMENTS}

A.A.Z., A.A.Z. Jr., and N.A.Z. would like to acknowledge support from the RFBR through Grants No. 14-02-00439 and No. 16-02-00556.
[1] A. Ashkin, Phys. Rev. Lett. 24, 156 (1970).

[2] M. P. Hughes and H. Morgan, J. Phys. D: Appl. Phys. 31, 2205 (1998).

[3] H. Morgan, M. P. Hughes, and N. G. Green, Biophys. J. 77, 516 (1999).

[4] D. J. Stevenson, F. Gunn-Moore, and K. Dholakia, J. Biomed. Opt. 15, 041503 (2010).
[5] N. K. Metzger, P. R. T. Jess, L. Paterson, E. M. Wright, and K. Dholakia, Proc. SPIE 5930, 59300M (2005).

[6] M. Ploschner, T. Čižmár, M. Mazilu, A. D. Falco, and K. Dholakia, Nano Lett. 12, 1923 (2012).

[7] K. Dholakia and P. Zemánek, Rev. Mod. Phys. 82, 1767 (2016).

[8] M. Ploschner, M. Mazilu, T. Čižmár, and K. Dholakia, Opt. Express 19, 13922 (2011). 
[9] V. Shvedov, A. R. Davoyan, C. Hnatovsky, N. Engheta, and W. Krolikowski, Nat. Photonics 8, 846 (2014).

[10] M. Dienerowitz, M. Mazilu, and K. Dholakia, J. Nanophotonics 2, 021875 (2008).

[11] A. A. Zharov, Jr., A. A. Zharov, I. V. Shadrivov, and N. A. Zharova, Phys. Rev. A 93, 013814 (2016).

[12] J. D. Jackson, Classical Electrodynamics (Wiley, New York, 1999).

[13] R. Zhao, P. Tassin, T. Koschny, and C. M. Soukoulis, Opt. Express 18, 25665 (2010).

[14] D. Van Thourhout and J. Roels, Nat. Photonics 4, 211 (2010).
[15] H. A. Pohl, Dielectrophoresis (Cambridge University Press, Cambridge, 1978).

[16] F. J. Rodríguez-Fortuño, N. Engheta, A. Martínez, and A. V. Zayats, Nat. Commun. 6, 8799 (2015).

[17] L. D. Landau and E. M. Lifshitz, Electrodynamics of Continuous Media (Pergamon Press, Oxford, 1984).

[18] M. Nieto-Vesperinas, J. J. Sáenz, R. Gómez-Medina, and L. Chantada, Opt. Express 18, 11428 (2010).

[19] S. B. Wang and C. T. Chan, Nat. Commun. 5, 3307 (2014).

[20] www.refractiveindex.info

[21] A. A. Zharov and V. V. Kurin, J. Appl. Phys. 102, 123514 (2007). 